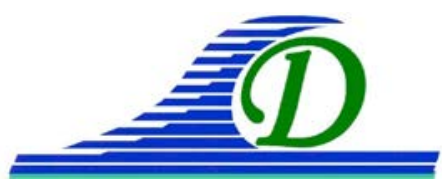
XIII ${ }^{\text {èmes }}$ Journées Nationales Génie Côtier - Génie Civil
Dunkerque, 2-4 juillet 2014

DOI:10.5150/jngcgc.2014.068 @ Editions Paralia CFL

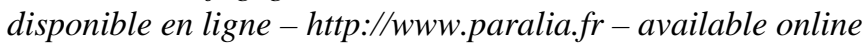

\title{
Apport de l'imagerie satellitaire pour le suivi du trait de côte
}

\section{Amélie ROCHE ${ }^{1}$, Christelle BOSC ${ }^{2}$, Laure CHANDELIER ${ }^{2}$, Renaud LAGNOUS ${ }^{2}$}

1. Cerema, Direction technique Eau mer et fleuves,

Technopôle Brest Iroise, 155, rue Pierre Bouguer, BP 5, 29280 Plouzané CEDEX, France.

Amelie.Roche@cerema.fr

2. Cerema, Direction territoriale Sud-Ouest, Pôle de compétences et d'innovation applications satellitaires et télécommunications (PCI AST)

Rue Pierre Ramond, CS 60013, 33166 Saint-Médard-en-Jalles CEDEX, France.

Christelle.Bosc@cerema.fr ; Laure.Chandelier@cerema.fr ;

Renaud.Lagnous@wanadoo.fr

\section{Résumé :}

Dans le cadre du Plan national d'Applications satellitaires et de la mise en œuvre de la Stratégie nationale de gestion intégrée du trait de côte, le Cerema (ex-CETMEF et exCETE SO) a réalisé un état de l'art des pratiques en matière d'identification et de suivi du trait de côte à partir d'images satellites. De nombreuses techniques existent, avec cependant des niveaux très variables d'automatisme, de complexité et de précision. Un prototype a été réalisé afin de tester et de quantifier les apports de l'imagerie satellitaire pour un suivi opérationnel du trait de côte. Exploitant des images Pléiades, il a été appliqué sur l'isthme de Miquelon-Langlade (Saint-Pierre-et-Miquelon, France) qui présente différentes géomorphologies : cordons dunaires, cordons de galets et secteurs anthropisés. Les résultats obtenus par 3 méthodes, exploitant sous différentes formes les informations contenues dans les canaux optiques (R, V, B, IR), ont été comparés à des mesures de terrain acquises à la même période. Les techniques de télédétection apportent des résultats satisfaisants, de précision métrique (erreur moyenne de $2 \mathrm{~m}$ ), sur les secteurs sableux, avec un indice lié à la limite de végétation. Cependant, l'erreur, inférieure à $5 \mathrm{~m}$, augmente en utilisant un indicateur de position du trait de côte différent ou en travaillant sur d'autres géomorphologies littorales. La méthode retenue sera prochainement testée sur un site de Saint-Pierre de type rocheux afin de valider les résultats et les utiliser pour un suivi annuel à grand rendement.

Mots-clés : Trait de côte, Images satellites, Suivi, Traitement automatique, Pléiades.

\section{Contexte et besoins}

L'évolution du littoral se traduit souvent par une analyse de mesures de terrain du "trait de côte" basées sur l'évolution de certains indicateurs du milieu liés à l'altimétrie, à l'hydrodynamique, à la géomorphologie ou à la botanique. Toutefois, ces mesures 
locales sont hétérogènes de par la diversité des méthodes et des outils de levé, la multiplicité des opérateurs, la variété des périodes de levé et parfois même la multiplicité des définitions du "trait de côte". Ainsi, elles ne permettent pas d'avoir une vision intégrée et nationale, ce que préconise depuis peu la "Stratégie nationale de gestion intégrée du trait de côte" (MEDDTL, 2012). En parallèle, l'imagerie spatiale déploie de plus en plus de satellites performants, tels que Pléiades, Spot 6 et 7 et bientôt Sentinel, offrant une résolution spatiale ou une revisite temporelle très élevées. Le développement d'outils libres, mettant à disposition des algorithmes éprouvés d'extraction de l'information des images, et le contexte politique, facilitant l'accès à la donnée avec la mise en place de plates-formes de mutualisation des données, rendent l'imagerie satellitaire de plus en plus abordable en termes de coût et de technicité pour des utilisations en lien avec les besoins sociétaux. S’inscrivant dans le cadre de ces politiques nationales, le Cerema (ex-CETMEF et ex-CETE SO) a conduit une étude sur l'apport potentiel de l'imagerie satellitaire pour la cartographie de traits de côte. Cet article présente d'abord l'état de l'art des pratiques en matière d'identification et de suivi de traits de côte à partir d'images satellites (BOSC et al., 2013); il aborde ensuite les résultats obtenus par un prototype réalisé sur Saint-Pierre et Miquelon afin de tester et de quantifier les apports potentiels de l'imagerie satellitaire pour un suivi opérationnel du trait de côte.

\section{Synthèse de l'état de l'art de l'exploitation des données satellitaires pour le suivi du trait de côte}

L'étude bibliographique (BOSC et al., 2013) a montré que la photo-interprétation était relativement simple à mettre en œuvre moyennant des images à très haute résolution (LE BERRE et al., 2005). Toutefois, elle est peu pertinente sur de larges territoires et pour des mises à jour fréquentes en raison du temps humain nécessaire à sa réalisation. Des méthodes de traitement semi-automatiques ont fait leurs preuves pour extraire le trait de côte défini comme la "limite d'immersion". Elles s'appliquent à la fois à des images optiques (GUERIN, 2001) ou radar (BAGHDADI et al., 2004). D’autres méthodes plus complexes, utilisant des classifications supervisées, permettent de cartographier les faciès littoraux (eau, végétation, sol nu, bâti, sable...) (EMRAN \& HAKDAOUI, 2003). Sur les côtes sableuses (MALLET et al., 2007 ; LAFON et al., 2010), le trait de côte défini comme le pied de dune est ainsi extrait avec une précision de l'ordre de 5 à $10 \mathrm{~m}$ suivant les images utilisées. Sur les côtes rocheuses (LAFON \& AUBIE, 2008), le trait de côte, défini comme le pied de falaise ou pied d'ouvrage, est parfois dissimulé par la présence d'ombres générées par les conditions d'acquisition de l'image, ce qui rend sa mesure plus délicate. Enfin, une méthode de traitement automatique a été mise au point afin de cartographier l'artificialisation du trait de côte, entendue comme reconnaissance automatique d'ouvrages (NASSER, 2011). 


\section{XIII ${ }^{\text {èmes }}$ Journées Nationales Génie Côtier - Génie Civil \\ Dunkerque, 2-4 juillet 2014}

Pour ces traitements, les images optiques sont intéressantes pour leurs richesses d'information mais ne sont pas exploitables lorsqu'elles génèrent des zones d'ombre ni en présence de couvert nuageux. Les images radar, exploitables par tout temps, de jour comme de nuit, peuvent être utilisées pour certains traitements (détection de la ligne d'immersion ou détection de zones d'humidité). Ces deux types d'images ont l'avantage de pouvoir couvrir des zones inaccessibles à l'opérateur et leur capacité de programmation présente un intérêt indéniable pour un suivi régulier ou post-événement. Les traitements automatiques offerts par la télédétection nécessitent un premier calage par rapport aux données de terrain et une évaluation de leur précision ; ils peuvent par la suite être utilisés en complément des suivis terrestres du trait de côte.

\section{Réalisation d'un prototype sur Saint-Pierre et Miquelon}

\subsection{Présentation du secteur étudié et des données disponibles}

Une scène d'images Pléiades a été acquise le $1^{\mathrm{er}}$ mai 2012 sur l'archipel de Saint-Pierreet-Miquelon. Les données mises à disposition via le programme Géosud sont des orthoimages 16 bits et 4 canaux (Rouge, Vert, Bleu et proche Infrarouge-IR-) d'une résolution de $50 \mathrm{~cm}$. Une zone de 1,5 par $5 \mathrm{~km}$ sur l'isthme de Miquelon-Langlande a été retenue comme zone-test, pour un linéaire d'environ $10 \mathrm{~km}$ de côte. L'isthme est en effet régulièrement suivi par les services de la Direction des territoires, de l'alimentation et de la mer (DTAM) en raison de phénomènes d'érosion pouvant provoquer la rupture de la seule route d'accès à Langlade. Le site est essentiellement sableux et dunaire, présentant également des cordons de galets et des secteurs anthropisés. Les traits de côte issus de différentes analyses de l'imagerie satellitaire ont ainsi pu être comparés aux résultats de terrain de la DTAM, réalisés en mai 2012. La précision des levés de la DTAM est supposée décimétrique en raison de la précision du levé DGPS couplée à l'interprétation par l'opérateur de la position de l'indicateur de trait de côte (voir figurés rouges figure 1). La précision géométrique de l'orthoimage Pléiades livrée par IGN Espace a, elle, pu être vérifiée par comparaison avec la BD Ortho® IGN sur 22 points de contrôle. L'exactitude planimétrique relative obtenue est de $1,3 \mathrm{~m}$, ce qui est en accord avec les précisions respectives annoncées sur la zone ( $1 \mathrm{~m}$ pour la BD Ortho ${ }^{\circledR}$ IGN et $0,8 \mathrm{~m}$ pour Pléiades). 


\section{Thème 3 - Instrumentation, mesures, imagerie et télédétection}
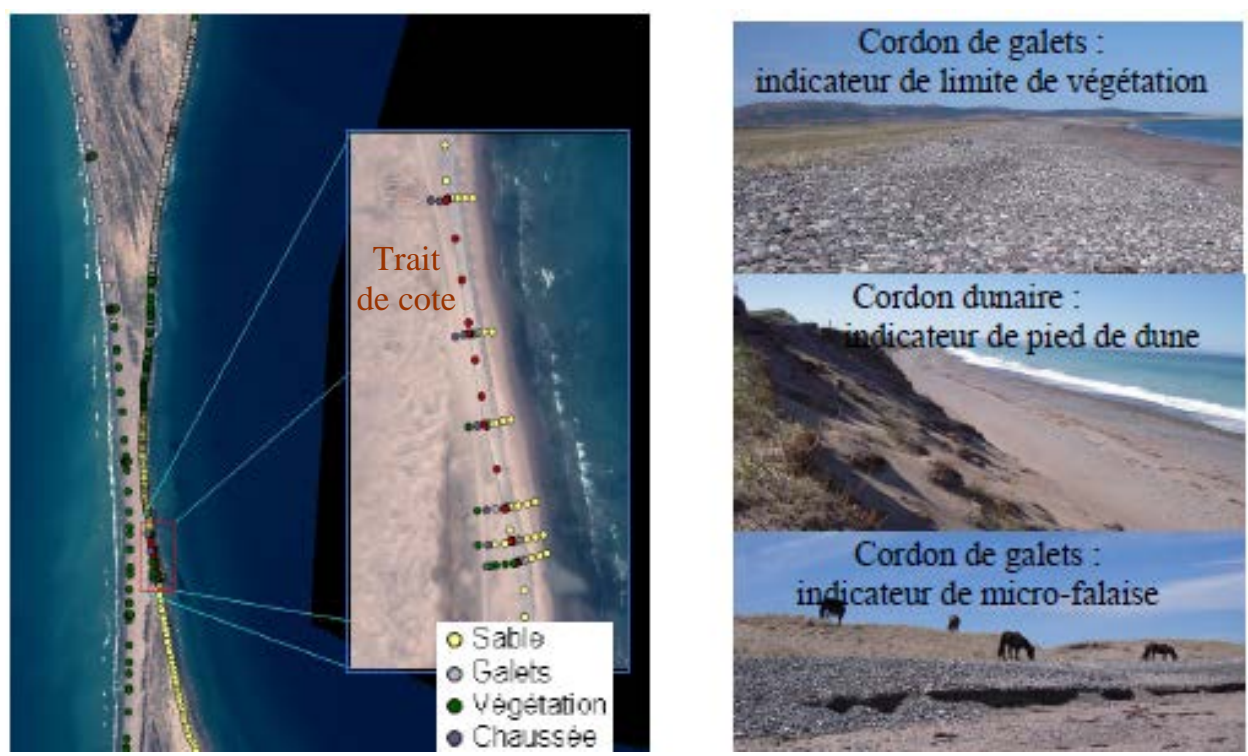

Figure 1. Zone d'étude avec types de géomorphologie et principaux indicateurs de trait de côte terrain levés par la DTAM (sources : CEREMA et DTAM975).

\subsection{Présentation de la méthodologie}

La méthodologie (LAGNOUS, 2013) procède en deux étapes : une classification de l'image afin d'obtenir une cartographie de faciès en 8 classes au regard de la géomorphologie du terrain et des indicateurs de trait de côte choisis par la DTAM puis, l'extraction automatique du trait de côte comme interface entre faciès "côté mer" et faciès "côté terre" (cf. nomenclatures des tableaux 1 et 2). Pour la phase de classification, qui conditionne grandement la qualité du résultat, trois méthodes utilisant l'algorithme SVM -séparateur à vaste marge- (BOSER et al., 1992) ont été testées. Des indices et des textures ont été calculés puis discriminés visuellement. Au final, 13 canaux ont été exploités : 4 canaux optiques, 2 indices (NDVI et GEMI) et 7 textures (l'énergie, l'entropie et la moyenne sur les canaux vert et proche IR ainsi que la texture high grey-level run emphasis sur le vert (OTB Developpment team, 2013)). Deux classifications supervisées par SVM ont été testées sur des nomenclatures à 8 et 25 classes correspondant à des analyses paysagères généralistes et détaillée. La multiplication des classes, censée améliorer le résultat, alourdit cependant la phase de préparation des données en augmentant le nombre d'échantillons d'apprentissage. Une troisième méthode adaptée d'un développement réalisé au PCI-AST pour l'occupation du sol (BOUFFIER \& HEBRARD, 2014) a été employée : une classification non supervisée par la méthode des k-moyennes (MACQUEEN, 1967) sur 8 classes définies "grossièrement" permet d'extraire automatiquement les différents types d'objets les composant, fournissant ainsi des échantillons d'entraînement plus fins et plus nombreux. L'algorithme SVM est alors appliqué sur ces sous-classes d'entraînement et le résultat est ensuite fusionné selon les 8 classes initiales. Dans la suite, seuls les 


\section{XIII ${ }^{\text {èmes }}$ Journées Nationales Génie Côtier - Génie Civil \\ Dunkerque, 2-4 juillet 2014}

résultats de cette troisième méthode, les plus prometteurs, sont présentés. Des posttraitements (filtre majoritaire principalement) sont effectués pour nettoyer la classification des pixels isolés. La dernière étape consiste à calculer la ligne séparant les classes de part et d'autre du trait de côte (extraction de contour sur une image binaire). Les traitements sont réalisés avec des logiciels libres: OrfeoToolBox (traitement des images satellite), QGIS et GRASS (vecteurs).

\subsection{Validation}

Le contrôle des classifications obtenues a été réalisé à partir d'échantillons de contrôle, avec des indicateurs de qualité classiques en télédétection (CALOZ \& COLLET, 2001) : (a) le coefficient kappa donne une mesure globale de qualité ; (b) les valeurs de F-Score fournissent des informations au niveau de chaque classe et (c) la matrice de confusion permet de voir, dans le détail, les commissions (pixels attribués à tort) et les omissions (pixels non détectés). Cette étude numérique a été combinée à une évaluation visuelle qui avait pour objectif de mieux cerner les zones ou faciès favorables ou non. La qualité du trait de côte extrait a été estimée en calculant sa distance à chaque point du levé terrain. Des cartographies de la précision du trait obtenu ont ainsi pu être produites et ont été complétées par une évaluation visuelle.

\section{Résultats obtenus et discussion}

La valeur de kappa obtenue pour la méthode donnant les résultats les plus satisfaisants est de 0,92 ce qui reflète un accord quasi-parfait sur les échantillons de contrôle. L'étude des F-Score et de la matrice de confusion (Tableaux 1 et 2) indique que les résultats de classification sont excellents pour les zones de végétation et pour l'eau. On observe de plus grandes difficultés dans les classes de plage et de galets pour lesquelles des confusions se produisent entre classes d'un même faciès mais de part et d'autre du trait de côte. Cette analyse numérique est confirmée par l'étude visuelle des résultats de classification (figure 2). Les secteurs anthropisés ne sont pas détectés: il s'agit d'éléments très isolés (quelques bâtiments), mal pris en compte. Concernant l'évaluation du trait de côte, en étudiant les écarts numériques avec les données terrain, on constate que le résultat diffère d'un indicateur à l'autre : les limites galets / galets conduisent à des résultats très variables : au nord-est, l'algorithme ne différencie pas les deux types de galets alors qu'au nord-ouest, sur des faciès équivalents, les résultats sont plus encourageants ; les limites sable / galet, à l'est, sont détectées de façon irrégulière ; les gabions sont très mal pris en compte; les limites de végétation sont très bien retrouvées (sauf sur une zone au centre ouest).

L'écart moyen calculé sur l'ensemble de la zone est de 5,3 m. Si on exclut les gabions et les crêtes de berme, cette valeur descend à $2,2 \mathrm{~m}$, ce qui, dans une zone où l'érosion peut être de $3 \mathrm{~m}$ par an (ROBIN, 2007), permet d'envisager une utilisation opérationnelle pour le suivi. 
Thème 3 - Instrumentation, mesures, imagerie et télédétection

Tableau 1. Valeur de F-Score

Tableau 2. Matrice de confusion

\begin{tabular}{ll}
\hline Classe & F-score \\
\hline Anthropisé & 0,00 \\
Végétation & 1,00 \\
Bas de plage & $\mathbf{0 , 8 5}$ \\
Plage & $\mathbf{0 , 5 9}$ \\
Haut de plage & 0,91 \\
Galets bas & $\mathbf{0 , 7 7}$ \\
Galet haut & 0,67 \\
Eau & 1,00 \\
\hline
\end{tabular}

Classes "côté Terre"

Classes "côté Mer"

\begin{tabular}{|c|c|c|c|c|c|c|c|c|}
\hline Classe & 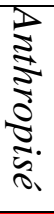 & 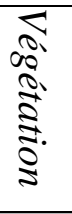 & $\begin{array}{l}\text { D } \\
\text { की } \\
\frac{0}{2} \\
\frac{2}{2}\end{array}$ & $\frac{\pi}{2}$ & $\begin{array}{l}\mathbf{I} \\
\stackrel{2}{\$} \\
\frac{0}{2} \\
\frac{2}{2}\end{array}$ & $\begin{array}{l}0 \\
0 \\
0 \\
\tilde{n} \\
0 \\
0 \\
\text { के }\end{array}$ & 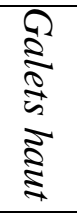 & $\stackrel{T}{\stackrel{T}{I}}$ \\
\hline Anthropisé & 0 & 22,1 & 0 & 0 & 77,9 & 0 & 0 & 0 \\
\hline Végétation & 0 & 100 & 0 & 0 & 0 & 0 & 0 & 0 \\
\hline Bas plage & 0 & 0 & 84,4 & 0,4 & 7,6 & 4,5 & 2,8 & 0,3 \\
\hline Plage & 0 & 0 & 0 & 88 & 11,9 & 0 & 0,1 & 0 \\
\hline Haut plage & 0 & 0 & 3,7 & 9,2 & 87,1 & 0 & 0 & 0 \\
\hline Galets bas & 0 & 0 & 0,2 & 2,3 & 0,4 & 78,3 & 18,8 & 0 \\
\hline Galets haut & 0 & 0 & 3,5 & 0,5 & 0,1 & 29,2 & 66,7 & 0 \\
\hline Eau & 0 & 0 & 0 & 0 & 0 & 0 & 0 & 100 \\
\hline
\end{tabular}
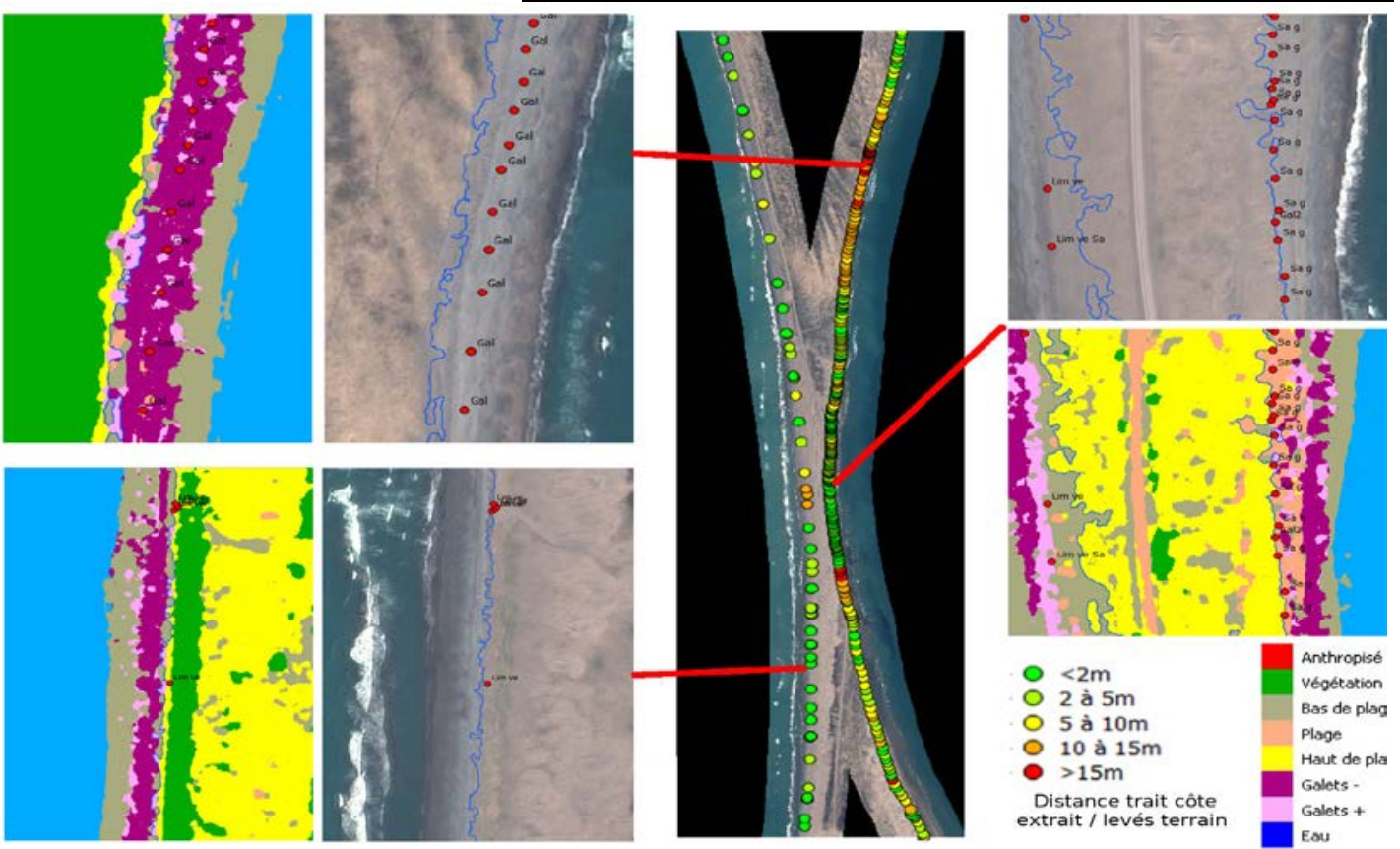

Figure 2. Résultats de la classification sur trois zones avec le trait de côte extrait et une vue d'ensemble avec la distance trait de côte / levés terrain.

\section{Conclusion et perspectives}

Le prototype réalisé sur l'isthme de Miquelon-Langlade obtient des résultats encourageants sur les secteurs sableux pour le suivi de la limite de végétation où la précision est métrique. Cependant, l'erreur augmente en utilisant un indicateur différent ou en travaillant sur d'autres géomorphologies (par exemple un cordon de galets). La performance de la méthode sera maintenant à éprouver sur d'autres types de côte et surtout dans le cadre d'un suivi temporel. En effet, la reproductibilité est un facteur 


\section{XIII ${ }^{\text {èmes }}$ Journées Nationales Génie Côtier - Génie Civil \\ Dunkerque, 2-4 juillet 2014}

essentiel pour que des comparaisons annuelles ou pluriannuelles soient possibles. Dans la méthode présentée ici, l'opérateur intervient principalement pour la définition des faciès composant le paysage et la saisie des échantillons d'apprentissage (étape allégée avec la chaîne de traitement PCI-AST) Ces opérations sont réutilisables, au moins partiellement, pour la mise à jour du trait de côte, le reste est réalisé automatiquement..

En ce qui concerne la méthodologie même, des pistes d'amélioration existent: amélioration de la fonction de passage de la carte des faciès au trait de côte, utilisation d'un modèle numérique de terrain, nouveaux indicateurs et comparaison des mouvements des faciès plutôt que du trait de côte seul (la cartographie disponible est plus riche que le trait).

La méthode présentant les meilleurs résultats sera prochainement testée sur l'île voisine de Saint-Pierre avec des images Pléiades de mai 2012 et 2013 afin de valider les résultats en absolu et de vérifier leur utilisation en relatif dans le cadre d'un suivi annuel à grand rendement. La nature de la côte sur cette nouvelle zone est principalement rocheuse, ce qui permettra d'évaluer la robustesse de l'algorithme lors d'un changement de paysage. Si les résultats sont probants, un outil automatique ou semi-automatique pourra être proposé à la DTAM pour l'accompagner dans son suivi du trait de côte, voire être généralisé à l'ensemble des côtes basses meubles ou rocheuses.

\section{Remerciements}

Le projet EQUIPEX GEOSUD, sélectionné parmi les "Équipements d’Excellence" du Programme Investissements d'Avenir (2011), vise à développer une infrastructure nationale de données satellitaires accessible gratuitement par la communauté scientifique et les acteurs publics. Il a permis l'acquisition des images Pléiades sur Saint-Pierre et Miquelon. La DTAM a gracieusement mis à disposition ses données de terrain pour le calage du prototype et la validation des résultats.

\section{Références bibliographiques}

BAGHDADI N., GRATIOT N., LEFEBVRE J.P., OLIVEROS C., BOURGUIGNON A. (2004). Coastline and mudbank monitoring in French Guiana: contributions of radar and optical satellite imagery. Canadian Journal of Remote Sensing, Vol. 30(2), pp 109-122. http://dx.doi.org/10.5589/m03-059

BOSC C., RAVIOLA M., LAINE P. (2013). Apport des technologies satellitaires pour la gestion du littoral : trait de côte et bathymétrie. Rapport CETMEF, $91 \mathrm{p}$.

BOSER B., GUYON I., VAPNIK V. (1992). A Training Algorithm for Optimal Margin Classifiers. $5^{\text {th }}$ Annual Workshop on Computational Learning Theory, pp 144-152, Pittsburgh, ACM.

BOUFFIER J., HEBRARD D. (2014). L'extraction d'informations à partir d'images satellites. Rapport MEDDE. 
CALOZ R., COLLET C. (2001). Précis de télédétection - Volume 3 : Traitements numériques d'images de télédétection. Presses de l'Université du Québec, Sainte Foy, Canada, 386 p.

EMRAN A., HAKDAOUI M. (2003). Suivi par télédétection de l'évolution spatiotemporelle de la frange littorale au nord-est de Tétouan. $2^{\text {nd }}$ FIG Regional Conference, Marrakech, Marocco, December 2-5, 2003, 9 p.

GUERIN (2011). Reconstruction bathymétrique de l'embouchure du bassin d'Arcachon à partir de l'analyse d'images satellites, Rapport de stage de Master 2 Recherche ENVOLH, Université de Bordeaux 1, 30 p.

LAFON V., AUBIE S. (2008). Cartographie des faciès géomorphologiques du littoral de la Côte Basque française à partir des données Formosat 2, BRGM/RP-56917-FR

LAFON V., HOAREAU A., MALLET C., DESPRATS J.F. (2010). Suivi du trait de côte en Aquitaine par imagerie Formosat-2, $\mathrm{XI}^{\text {èmes }}$ journées Nationales Génie Côtier Génie Civil, Les Sables d’Olonne, pp 497-504. http://dx.doi.org/10.5150/jngcgc.2010.058-L

LAGNOUS R. (2013). Évaluation de l'apport de l'imagerie satellitaire pour le suivi du trait de côte. Rapport de Master 2 "Surveillance et gestion de l'environnement", Université Paul Sabatier, Toulouse 3, 34 p.

LE BERRE I., HENAFF A., DEVOGELE T., MASCRET A., WENZEL F. (2005). Spot 5 : un outil pertinent pour le suivi du trait de côte ? Norois. http://dx.doi.org/10.4000/norois.378 MACQUEEN J. (1967). Some Methods for classification and Analysis of Multivariate Observations. Proceedings of Fifth Berkeley Symposium on Mathematical Statistics and Probability, Vol. 1, pp 281-297, University of California Press.

MALLET C., LAFON V., DESPRATS J.F. (2007). Cartographie des faciès géomorphologiques du littoral aquitain à partir de l'imagerie spatiale Formosat-2, BRGM/RP-56101-FR

MEDDTL (2012). Stratégie nationale de gestion intégrée du trait de côte: vers la relocalisation des activités et des biens. MEDDTL, 20 p. Disponible en ligne sur URL: http://www.developpement-durable.gouv.fr/Strategie-nationale-de-gestion.html

NASSER G. (2011). Méthodologie de traitements d'images en réponses à des besoins thématiques exprimés par des acteurs de la thématique Mer et Littoral. CNES. Rapport de Master2 "Techniques spatiales et instrumentation", Université Paul Sabatier, 38 p.

OTB DEVELOPMENT TEAM (2013). The Orfeo ToolBox Cookbook, a guide for nondevelopers. Disponible en ligne sur URL : http://www.orfeo-toolbox.org/CookBook/

ROBIN N. (2007). Morphodynamique des systèmes de flèches sableuses - Étude comparative entre les embouchures tidales de l'Archipel de Saint Pierre et Miquelon et de la côte Ouest du Cotentin (Manche). Rapport de synthèse. Université de Caen, Morphodynamique Continentale et Côtière, CNRS-UMR 6143, 26 p. Disponible en ligne sur URL :http://www.dune-miquelon-langlade.net/files/file/Etude_sedimentaire.pdf 